
allemande

49-2 | 2017

L'année 1917, entre ancien et nouveau monde

\title{
Les relations gréco-allemandes au cours de l'année 1917
}

Elli Lemonidou

\section{(2) OpenEdition}

9 Journals

Édition électronique

URL : https://journals.openedition.org/allemagne/578

DOI : 10.4000/allemagne.578

ISSN : 2605-7913

Éditeur

Société d'études allemandes

Édition imprimée

Date de publication : 29 décembre 2017

Pagination : 369-380

ISSN : 0035-0974

\section{Référence électronique}

Elli Lemonidou, « Les relations gréco-allemandes au cours de l'année 1917 », Revue d'Allemagne et des pays de langue allemande [En ligne], 49-2 | 2017, mis en ligne le 29 décembre 2018, consulté le 21 mai 2021. URL : http://journals.openedition.org/allemagne/578 ; DOI : https://doi.org/10.4000/allemagne 578 


\section{Les relations gréco-allemandes au cours de l'année 1917}

\section{- Elli Lemonidou*}

L'année 1917 fut non seulement un jalon très important du développement de la Première Guerre mondiale et de l'histoire de l'Europe en général, mais elle fut également cruciale en ce qui concerne le rôle et la participation de la Grèce dans le grand conflit. Les relations germano-grecques en 1917 - et au cours, au total, des deux dernières années de la guerre - peuvent être examinées selon les axes suivants:

- Le réseau politique et diplomatique des relations bilatérales, qui concerne notamment la question du maintien ou non de la neutralité de la Grèce, en raison de la pression croissante exercée sur elle par les forces de l'Entente pour obtenir son alliance, jusqu'à la résolution finale de la question à l'été 1917.

- La réalité militaire avec la présence des troupes germano-bulgares sur le front de Macédoine: une partie importante de ces troupes se trouvait très proche de la frontière nord de la Grèce, tandis qu'une autre avait déjà, depuis l'été 1916, occupé une partie considérable de la Macédoine orientale. Cette réalité se trouva liée à une série d'attentes (réalistes ou pas) de chaque côté et fut inextricablement mêlée, entre autres, à l'évolution de l'alliance entre l'Allemagne et la Bulgarie.

- Le séjour du IV e corps d'armée grec dans la ville allemande de Görlitz après sa capture à l'automne 1916, ce qui ajouta une nouvelle dimension aux relations entre les deux pays. Le régime spécifique appliqué aux militaires grecs eut en effet pour résultat, comme nous le verrons, que ces derniers purent, d'une part, constituer un instrument de négociation, d'autre part, entrer en osmose avec la communauté locale ${ }^{(1)}$.

* Docteur en histoire des relations internationales, professeure assistante à l'Université de Patras.

1 Une grande partie du matériel utilisé pour le présent travail provient de mes recherches dans les archives et bibliothèques de Stuttgart, recherches que j'ai pu effectuer à l'automne et à l'hiver 2015 grâce au programme européen CENDARI. Je souhaite adresser mes vifs remerciements aux responsables du programme, au professeur Gerhard Hirschfeld, éminent spécialiste de la Première Guerre mondiale, ainsi qu'à Irina Renz, Christian Westerhoff et Dennis Grinat pour toute leur aide précieuse sur place, à Stuttgart. 


\section{Les relations bilatérales gréco-allemandes}

Les évolutions décisives de l'année 1917 en Grèce ont leur source dans l'été de l'année précédente, lorsque les forces germano-bulgares décidèrent de passer à l'action sur le front macédonien. Jusqu'à ce moment, Berlin avait évité d'entreprendre une action contre l'armée d'Orient (arrivée sur le sol grec depuis le mois d'octobre 1915) par peur d'indisposer ses alliés turcs et surtout bulgares. Aux demandes pressantes en faveur d'une offensive commune en Macédoine présentées par le roi de Grèce et les chefs de l'armée grecque qui, depuis le début de la guerre, se trouvaient en négociations permanentes et secrètes avec l'Allemagne, cette dernière opposait la non-préparation des troupes germano-bulgares ${ }^{(2)}$. C'est seulement en août 1916, et pour répondre à l'avancée des forces de l'Entente au-delà du Strymon, que les forces germano-bulgares entreprirent une double attaque sur les deux ailes du front macédonien. Sur le côté ouest, l'attaque conduisit à l'occupation de la ville de Florina pour une période d'environ un mois tandis que, sur le côté est, les troupes surtout bulgares (les forces allemandes étaient toujours moins nombreuses) avancèrent en Macédoine orientale, occupant Serrès, Drama et Cavala. Durant l'occupation de ces villes, les Bulgares se montrèrent particulièrement cruels, massacrant et terrorisant les populations locales, et cela malgré les assurances contraires qui avaient été données au roi de Grèce ${ }^{(3)}$. L'armée grecque ne put résister et c'est dans cette situation extrême que s'ouvrit une page tragique de son histoire, avec l'entrée en captivité du IV corps, le 11 septembre.

La livraison de la Macédoine orientale à l'ennemi héréditaire qu'était la Bulgarie fut, comme cela était prévisible, lourde de conséquences pour l'opinion publique grecque $^{(4)}$ et joua également un rôle de catalyseur sur la scène politique. C'est à cause de cette avance germano-bulgare sur le sol grec qu’Élefthérios Venizélos prit la décision de se rendre à Salonique afin d'y créer un gouvernement provisoire et mettre sur pied une armée qui se battrait aux côtés de l'armée d'Orient, afin de sauver l'honneur national. C'est aussi en raison de cette avance que les Alliés décidèrent d'aider le gouvernement nouveau-né de Venizélos, tout en durcissant leur politique à l'égard du roi et de son entourage. Les relations entre Alliés et Athènes se dégradèrent de jour en jour, le point culminant de cette tension se plaçant dans les premiers jours de décembre 1916 avec les événements appelés «Noemvriana» (qui signifie «de novembre», à cause du calendrier grégorien qui était alors en vigueur en Grèce), c'est-

2 Bundesarchiv Militärarchiv - Fribourg-en-Brisgau (BA MA), RM 5/2425, télégramme très confidentiel n² 278 du 16 octobre 1916, envoyé d'Athènes au ministère des Affaires étrangères allemand et Mirbach, ministre allemand à Athènes, à Falkenhayn, chef de l'état-major allemand, télégramme $\mathrm{n}^{\circ} 397$ du 25 juin 1916. Georges B. Leon (Leontaritis), Greece and the Great Powers. 1914-1917, Thessalonique, Institute for Balkan Studies, 1974, p. 352-364.

3 G. B. Leon (Leontaritis), Greece and the Great Powers (note 2), p. 380-389, 396-400; Hellenic Army General Staff (Army History Directorate), A Concise History of the Participation of the Hellenic Army in the First World War. 1914-1918, Athènes, Hellenic Army General Staff, 1999, p. 79-91.

4 BA MA, RM 5/2425, Falkenhausen, attaché militaire allemand à Athènes, à Falkenhayn, télégramme

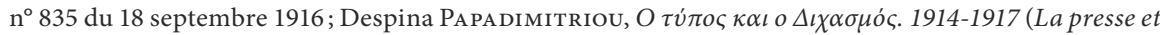
la Discorde. 1914-1917), thèse de doctorat non publiée, Athènes, Université d'Athènes, 1990, p. 117-125 et $134-140$. 
à-dire les conflits armés entre soldats grecs et soldats alliés survenus en plein centre d'Athènes qui firent de nombreux morts et blessés de part et d'autre ${ }^{(5)}$. À partir de ce moment, les pays de l'Entente ne cessèrent d'avoir recours à des mesures de coercition à l'encontre de la Grèce royaliste. La première d'entre elles fut un blocus naval qui commença le 8 décembre au matin et se prolongea pendant six mois, entraînant la dégradation générale de la vie des Grecs. Les objectifs de cette politique étaient l'élimination du roi Constantin et l'implication de la Grèce dans le conflit aux côtés des Alliés. Ils furent atteints lors des conférences alliées de Paris et de Londres qui eurent lieu en mai $1917^{(6)}$ et eurent pour résultat, en juin 1917, le détrônement de Constantin, la prise du pouvoir par Venizélos et l'entrée en guerre de la Grèce aux côtés de l'Entente.

Quant aux Allemands, ils suivaient toutes ces évolutions si décisives pour la Grèce comme simples observateurs, sans intervenir. Leur préoccupation première était de ne pas indisposer leurs alliés bulgares qui, lassés par la guerre, pouvaient à tout moment abandonner l'effort commun et signer une paix séparée avec le camp adverse. D’une certaine façon, le détrônement de Constantin et l'entrée en guerre de la Grèce libéraient le gouvernement de Guillaume II, en lui permettant de se prononcer sur le sort des régions grecques tant convoitées par les Bulgares. C'est ainsi qu'à la fin du mois de juillet 1917, les gouvernements allemand et austro-hongrois informèrent Sofia qu'ils ne s'opposeraient pas à l'occupation de la Macédoine orientale par la Bulgarie et à l'annexion des villes de Cavala, Serrès et Drama qui se trouvaient déjà sous occupation bulgare $^{(7)}$. Le seul qui continuait à rêver d'une attaque germano-bulgare sur le front macédonien était l'ancien roi Constantin qui, après son exil en Suisse, proposa à Berlin des plans d'attaque de la Grèce, attaque dont il espérait qu'elle pourrait entraîner une révolution, le renversement du cabinet de Venizélos et sa propre réhabilitation. Or, la Macédoine orientale étant déjà promise à la Bulgarie, tout projet de ce type était contraire aux intérêts allemands, et donc voué à l'échec ${ }^{(8)}$. L'éventualité d'une attaque allemande contre l'armée d'Orient avec la contribution de la Grèce fut de nouveau

5 Service historique de la Marine nationale - Vincennes (SHM), SS X f 9, Roquefeuil à Lacaze, «Rapport sur les événements qui se sont déroulés dans les premiers jours de décembre 1916 », n 533 du 9 décembre 1916; Léon MaccAs, «Les événements d'Athènes des $1^{\text {er }}$ et 2 décembre 1916 », Revue des Deux Mondes, tome XXXVIII (mars-avril 1917), p. 96-135; Louis DARTIGE Du Fournet, Souvenirs de guerre d'un amiral. 1914-1916, Paris, Plon, 1920, p. 210-273; Georges Boussenot, «Le Drame du $1^{\text {er }}$ décembre 1916 à Athènes", Revue d'Histoire de la Guerre Mondiale, nº 1 (janvier 1938), p. 1-27; Yannis MouréLos, L'intervention de la Grèce dans la Grande Guerre (1916-1917), Athènes, Institut français d'Athènes,

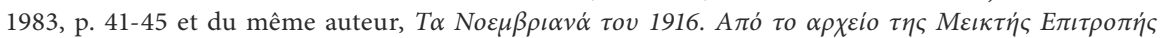

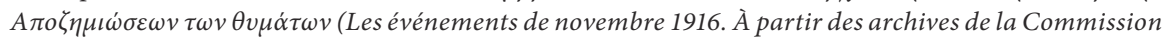
Mixte des Indemnités des victimes), Athènes, Patakis, 2007.

6 Archives du ministère des Affaires étrangères de France - Paris (MAE), 994, Conférence de Paris des 4 et 5 mai 1917, procès-verbaux; MAE, 994, Conférence de Londres des 28 et 29 mai 1917, procès-verbaux.

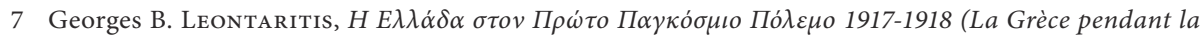
Première Guerre mondiale 1917-1918), Athènes, MIET, 2000, p. 117-118, 125-127.

8 Landesarchiv Baden-Württemberg, Hauptstaatsarchiv Stuttgart (HStAS), Q 1/18 Bü 149, rapport très confidentiel intitulé «Bericht über die Besprechungen des Königs und der Königin von Griechenland mit Professor D. Jäckh in St. Moritz am 5. September 1917 »; G. B. LeOnTARITIS, La Grèce pendant la Première Guerre mondiale (note 7), p. 127-138. 
envisagée par Guillaume II lui-même dans les premiers mois de 1918, mais ces projets se heurtèrent aux objections du ministère des Affaires étrangères et de la direction suprême de l'état-major ${ }^{(9)}$.

Sur le plan politique et diplomatique, ce furent alors les Alliés qui eurent l'initiative, avec l'affaiblissement continu du rôle de l'Allemagne. Et pourtant, en Grèce même, la cause allemande recueillait de plus en plus de sympathies, même si la germanophilie était plutôt une caractéristique du camp royaliste. Le roi lui-même, beau-frère de Guillaume II, éprouvait de l'admiration pour l'Allemagne et, pour une grande partie de la population, la fidélité à Constantin était liée à la fidélité à l'Allemagne. Outre le souverain et la cour, des hommes politiques et des universitaires éprouvaient des sentiments germanophiles, de même qu'une grande partie des officiers supérieurs de l'armée grecque, formés à l’Académie militaire de Berlin; le chef et le sous-chef de l'état-major grec étaient convaincus que les Allemands seraient victorieux ${ }^{(10)}$.

La propagande allemande en Grèce, très bien organisée dès le début du conflit, contribua à l'évolution des consciences. Elle était dirigée par l'ambassadeur d'Allemagne à Athènes, le comte Wilhelm von Mirbach. Celui-ci fut secondé dans son travail par un agent spécial délégué aux affaires de presse, le fameux baron Karl Freiherr von Schenck, ancien directeur de l'agence Wolff, qui était arrivé en Grèce avant la guerre comme représentant de la maison Krupp. Les deux hommes dépensaient des sommes énormes en subventions à la presse athénienne et de province afin d'acheter des sympathies et des consciences parmi les journalistes grecs. L'objectif principal de la propagande allemande était de mettre l'accent sur la puissance et l'invincibilité de l'armée du Reich et ainsi de diffuser la peur au sein de la population grecque, de même que le renoncement à la participation à la guerre aux côtés de l'Entente ${ }^{(11)}$. Face aux considérables efforts allemands, la propagande des Alliés manqua tout au long de la guerre de moyens, d'organisation et de coordination ${ }^{(12)}$. Cela eut pour effet d'orienter de plus en plus l'opinion grecque vers l'Allemagne

9 L’Allemagne et les problèmes de la paix pendant la Première Guerre mondiale, vol. IV : 4 mars-4 octobre 1918, documents réunis par André Scherer et Jacques Grunewald, Paris, Publications de la Sorbonne, 1978, p. 19-21: Grünau à Hertling, télégramme nº 51 du 10 mars 1918; G. B. LeOntaritis, La Grèce pendant la Première Guerre mondiale (note 7), p. 138-145.

10 MAE, 244, Braquet à Millerand, rapport nº 13 du 11 février 1915; Georges M. MÉLAs, L'ex-roi Constantin. Souvenirs d’un ancien secrétaire, Paris, Payot, 1921, p. 195-209; Kostas Loulos, "Oı $\varepsilon \lambda \lambda \eta v o-$

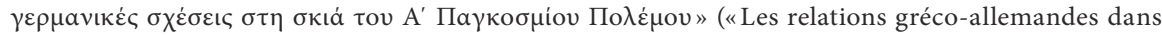
l'ombre de la Première Guerre mondiale»), in: Evangelos Chrysos, Wolfgang Schultheiss (dir.),

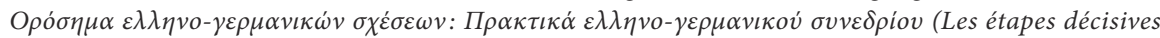
des relations gréco-allemandes), Athènes, Fondation du Parlement des Grecs pour le Parlementarisme et la Démocratie, 2010, p. 172-176.

11 MAE, 257, Astraud à Roques, rapport s.n. du 10 juin 1916; Jean-Claude MontAnt, «Aspects de la propagande française en Grèce», in: La France et la Grèce dans la Grande Guerre, actes du colloque tenu en novembre 1989 à Thessalonique, Université de Thessalonique, 1992, p. 63.

12 Gabriel Deville, L'Entente, la Grèce et la Bulgarie. Notes d'histoire et souvenirs, Paris, Eugène Figuière, 1919, p. 182; G. M. MÉLAs, L’ex-roi Constantin (note 10), p. 31-32; Elli Lemonidou, La Grèce vue de France pendant la Première Guerre mondiale; entre censure et propagandes, thèse de doctorat non publiée, Paris, Université Paris IV-Sorbonne, 2007, p. 41-45. 
et de la détourner de la francophilie incontestable des débuts du conflit ${ }^{(13)}$. L'attachement à l'Allemagne se renforça encore avec la politique autoritaire des Alliés, marquée par le blocus ${ }^{(14)}$ et le détrônement de Constantin, détrônement qui, pour une grande partie des Grecs, signifia la perte de leur liberté et leur asservissement à l'Entente ${ }^{(15)}$.

\section{Sur le front macédonien}

Comme il ressort clairement de toutes les sources disponibles, de l'époque et postérieures, l'avancée des forces des Puissances centrales, surtout après les succès de l'automne 1915 aboutissant à la défaite et à l'expulsion de l'armée serbe, en combinaison avec le débarquement des troupes de l'Entente à Thessalonique, conduisit à une raideur stratégique, qui allait être maintenue jusqu'à l'automne 1918. Dans ce contexte, une question clé pour la planification stratégique et l'action militaire des Empires centraux était de savoir à quel point une avance des troupes allemandes vers le sud, ayant pour but de chasser les forces de l'Entente et d'occuper Thessalonique, était souhaitable et réalisable. Cette perspective créait également de grandes attentes dans la communauté allemande qui vivait à Thessalonique et qui était de taille importante ${ }^{(16)}$. Il fut cependant assez vite évident qu'un certain nombre de raisons politiques et militaires rendaient cet objectif illusoire $^{(17)}$. Au niveau politique et diplomatique, l'avance allemande risquait de provoquer des gains territoriaux que la Bulgarie pourrait revendiquer, entraînant l'accroissement du ressentiment de la Grèce à son égard. Du côté militaire, le problème posé était celui du ravitaillement des forces germano-bulgares, d'autant plus que le réseau ferroviaire existant dans la région était extrêmement limité(18).

Comme nous l'avons vu, une première évolution cruciale eut lieu à l'été 1916. L'occupation d'une partie de la Macédoine orientale par les troupes germano-bulgares, la captivité du IV corps d'armée grec et son transport à Görlitz contribuèrent de façon décisive au renforcement des forces antimonarchiques, forces qui accusèrent ouvertement la politique menée par le roi Constantin de conduire à une diminution du territoire national. Le point culminant de cette évolution fut la formation du gouvernement

13 Service historique de la Défense - Vincennes (SHD), 7 N 1337, Braquet à Millerand, rapport $n^{\circ} 8$ du 2 février 1915; MAE, 284, Guillemin à Briand - de la part de Braquet pour Gallieni, télégramme $\mathrm{n}^{\circ} 707$ du 10 novembre 1915; G. MÉLAs, L’ex-roi Constantin (note 10), p. 151.

14 SHD, 1340, Braquet à Painlevé, télégramme nº 145 du 6 novembre 1917; BA MA, RM 5/2754, rapport confidentiel rédigé à Berlin, nº 16837 B II du 8 juin 1918; E. Lemonidou, La Grèce vue de France (note 12), p. 321-329.

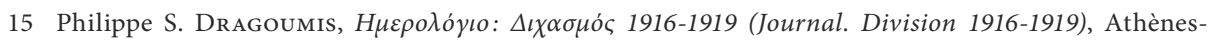
Ioannina, Dodoni, 1995, p. 21-29.

16 Max Brunau, Das Deutschtum in Mazedonien, Stuttgart, Ausland und Heimat Verlags-Aktiengesellschaft, 1925, p. 45. L'auteur souligne qu'une telle perspective aurait probablement reçu un accueil favorable dans une partie de la population grecque qui souhaitait un contrôle des troupes anglo-françaises occupant l'arrière-pays grec. Il est à noter que la communauté allemande de Thessalonique s'est considérablement réduite à partir de l'occupation de la ville par les troupes de l'Entente (p. 45-48).

17 Schlachten des Weltkrieges (im Auftrage des Reichsarchivs), Bd. 11: Weltkriegsende an der mazedonischen Front, bearbeitet von D. Dieterich, Berlin, Oldenburg, 1925, p. 9.

18 Der Weltkrieg 1914 bis 1918. Die militärischen Operationen zu Lande, Bd. 11: Die Kriegsführung im Herbst 1916 und im Winter 1916/17, Berlin, Mittler, 1938, p. 338. 
de Thessalonique, avec le soutien des forces de l'Entente qui augmentèrent en même temps leur pression sur Constantin. De leur côté, les dirigeants des Puissances centrales gardaient l'espoir que cette attitude particulièrement interventionniste des puissances de l'Entente pourrait avoir un effet inverse et pousser la Grèce à mettre fin à sa neutralité et à entrer officiellement dans la guerre aux côtés des Allemands ${ }^{(19)}$.

La conclusion qu'en 1917 une telle perspective semblait désormais impossible, surtout après les événements sanglants de décembre 1916 à Athènes et la pression dramatique exercée par le blocus allié sur la population grecque, en combinaison avec la nouvelle situation que créait, pour le front macédonien, la reprise de Monastir par les forces du général Sarrail, commandant de l'armée d'Orient, créait une situation qui exigeait une révision générale de la stratégie sur le front de Macédoine. Le commandement bulgare surtout commençait à subir une pression intense pour confirmer sa capacité d'obtenir des gains territoriaux substantiels d'ici la fin des hostilités. Lors d'une réunion tenue dans la ville de Pleß en janvier 1917, l'Allemagne confirma qu'elle ne pouvait engager davantage de forces sur le front macédonien, tandis qu'il fut souligné une fois de plus qu'une action offensive en Macédoine n'était pas possible sans une attaque parallèle de l'armée grecque au sud ${ }^{(20)}$.

La perspective d'une attaque sur le front macédonien étant ainsi exclue en 1917, la lassitude et la réflexion gagnèrent le camp bulgare, tandis que, selon les conservateurs, les Bulgares devaient avant tout préserver leurs acquis et limiter leurs revendications supplémentaires à la récupération de Monastir et à l'occupation de Thassos ${ }^{(21)}$. Dans le même temps, aussi bien du côté de l'Entente que des Puissances centrales, il était maintenant clair que l'enjeu principal du maintien des deux adversaires dans l'espace macédonien était la consolidation défensive et la possibilité de fixer l'adversaire, afin que ses troupes ne puissent être employées sur d'autres fronts de guerre ${ }^{(22)}$. Au printemps 1917, les Puissances centrales purent gravement perturber, grâce à l'action des sous-marins allemands en Méditerranée orientale, le ravitaillement en munitions de l'armée d'Orient ${ }^{(23)}$. Mais cela ne changea rien à la stratégie allemande. Le retrait du roi Constantin et le retour au pouvoir de Venizélos constituèrent une source de préoccupation légitime au sein des états-majors germano-bulgares, préoccupation qui fut cependant modérée par la certitude que Venizélos n'était pas en mesure de former et de mettre rapidement à la disposition de l'Entente une force militaire en état de combattre ${ }^{(24)}$.

Pendant ce temps, la situation sur le front macédonien demeurait presque inchangée, marquée seulement par des événements mineurs ${ }^{(25)}$. La stagnation sur le front

19 Ibid., p. 345. Voir aussi Carl MüHLMAnN, Oberste Heeresleitung und Balkan, Berlin, Wilhelm-Limpert Verlag, 1942, p. 183-188.

20 Der Weltkrieg 1914 bis 1918. Die Kriegsführung im Herbst 1916 und im Winter 1916/17 (note 18).

21 Der Weltkrieg 1914 bis 1918. Die militärischen Operationen zu Lande, Bd. 12: Die Kriegsführung im Frühjahr 1917, Berlin, Mittler, 1939, p. 520. Ibid., p. 522-523; C. Mühlmann, Oberste Heeresleitung und Balkan (note 19), p. 188-189. Der Weltkrieg 1914 bis 1918. Die Kriegsführung im Frühjahr 1917 (note 21), p. 525. Ibid., p. 526. plus grande partie de Thessalonique en août 1917. Le témoignage du pasteur Brunau (note 16) est à 
et la fatigue croissante de tous les protagonistes, qui se manifestait de différentes manières dans chaque camp, soulevaient des préoccupations encore plus grandes dans le camp bulgare, d'autant plus qu'à l'automne 1917 les rumeurs selon lesquelles l'Allemagne allait négocier une paix sans modifications territoriales s'intensifiaient ${ }^{(26)}$. Or, cette perspective n'allait guère dans le sens d'un engagement bulgare aux côtés des Puissances centrales. L'attitude de la Bulgarie dans la guerre et, surtout, au cours de l'offensive décisive menée par l'Entente en septembre 1918 fait partie des questions importantes abordées par l'historiographie allemande de la Première Guerre mondiale, avec des interprétations qui varient en fonction des périodes, des auteurs et des sources disponibles. Dans ce contexte, les mémoires de ceux qui vécurent de près la situation sur le front macédonien confirment le sentiment de stagnation qui l'emportait, avec une alternance de journées d'inactivité complète et de journées marquées par des actions de petite envergure. Ils montrent aussi l'incertitude à propos d'une offensive vers le sud, mais aussi le sentiment de la spécificité du front qu'ils observaient: selon eux, celui-ci était considéré comme inférieur en importance alors qu'il présentait de nombreuses caractéristiques propres et porteuses de danger, tout autant que les deux grands fronts d'Europe occidentale et orientale ${ }^{(27)}$.

\section{Les Grecs de Görlitz}

Dans le même temps, à plusieurs kilomètres du front, la présence du IV e corps d'armée grec à Görlitz ouvrait un nouveau chapitre des relations gréco-allemandes, un chapitre en partie lié à la guerre, mais qui s'en distinguait aussi par de nombreux traits. Le déplacement d'une force militaire significative d'un pays neutre sur le territoire d'un autre État, avec les spécificités des troupes grecques de Görlitz, constitue certainement l'une des pages très particulières de l'histoire de la Première Guerre mondiale, page qui, depuis plusieurs décennies, semble avoir été oubliée en Grèce comme en Allemagne. C’est seulement très récemment qu'un effort d'enquête historique

cet égard éclairant: Brunau avait une très bonne connaissance de la ville et fut en contact à plusieurs reprises avec les forces de l'Entente qui en étaient alors les maîtres. Dans un texte ultérieur, il met d'ailleurs en cause l'attitude des Français: non seulement ils ne firent rien pour enrayer l'incendie, mais ils participèrent en outre au pillage des commerces de la ville.

26 Schlachten des Weltkrieges (note 17), p. 15-16.

27 Le témoignage de Erich von Schede est ici particulièrement utile. Schede servit comme médecin dans l'armée allemande sur le front macédonien et consigna son expérience dans des lettres ou dans son journal. Ses notes furent publiées après la guerre: Dr. Med. (Erich von) ScHede, Als Arzt in Mazedonien 1916-1918. Briefe und Betrachtungen eines Arztes, Nordhausen a. Harz, Verlag von L. Hornickels Buchhandlung, 1929. Bien que le volet médical de ces notes ne soit pas directement lié à la dimension grecque de la guerre ou aux relations gréco-allemandes, elles sont cependant d'un grand intérêt pour la description de la vie quotidienne au front, avec l'évocation des conditions difficiles en termes de matières premières et de logistique (p. 33) ou de l'immobilité prolongée (p. 68), mais aussi pour la mise en valeur de la spécificité du front et le fait que l'opinion publique en avait une connaissance limitée; cette dernière caractéristique provoqua d'ailleurs en grande partie la décision de Schede d'enregistrer en continu ce qu'il voyait (p. 54). Schede évoque également les projets d'infrastructure entrepris par l'armée allemande dans la région du front (p. 62), formule de temps en temps des espoirs d'une paix rapide (p. 27 et 62) et s'intéresse à l'effet sur les troupes des évolutions positives du conflit pour l'Allemagne, telles que les succès de la guerre sous-marine (p. 34) ou le cessez-le-feu avec la Russie (p. 76). 
systématique sur la question a été lancé, au-delà des approches émotionnelles ou politiquement orientées qui, en Grèce, ont dominé pendant de nombreuses années dans le regard sur l'événement ${ }^{(28)}$.

L'histoire du transfert des Grecs à Görlitz commence en septembre 1916. Le IV corps d'armée grec se trouva pris au piège dans une impasse que composaient: a) l'avance des forces germano-bulgares dans l'est de la Macédoine, avec la présence d'une majorité de forces bulgares, présence qui causait une grande inquiétude dans la population grecque de la région ${ }^{(29)}$; b) l'approche parallèle des forces navales de l'Entente près de Cavala; c) la pression du mouvement vénizéliste de Thessalonique; d) la réticence du gouvernement royal d'Athènes à s'engager dans des hostilités contre les troupes des Puissances centrales; e) de graves problèmes administratifs à un moment très sensible, la communication avec Athènes étant devenue particulièrement difficile.

Dans ce contexte étouffant, le colonel Ioannis Chatzopoulos, qui faisait office de commandant du corps, pris en tenaille entre l'impossibilité matérielle d'une résistance armée contre les agresseurs et une série de choix douloureux (dont le pire aurait été, pour lui et ses hommes, la captivité aux mains des Bulgares), adressa au général Hindenburg, commandant en chef des forces allemandes, une demande de transport et d'hébergement du IV corps grec sur le territoire allemand jusqu'à la fin de la guerre. La demande fut acceptée, prenant même en compte presque tous les souhaits exprimés par Chatzopoulos. Quelques jours plus tard, officiers et soldats commençaient leur long voyage vers Görlitz.

Le retentissement de la nouvelle fut particulièrement intense en Grèce, tant au moment où les événements se produisirent que dans les années qui suivirent, constituant une épine de plus dans les relations extrêmement tendues entre le camp royaliste et le camp vénizéliste. Le retentissement fut également intense au niveau internatio$\mathrm{nal}^{(30)}$. L'Allemagne souhaitait démontrer à tous qu'elle réservait un bon accueil au corps militaire d'un pays neutre. D’après les témoignages de l'époque, la réception des militaires grecs après leur pénible voyage peut être qualifiée d'impressionnante, avec la participation massive des habitants et la présence d'un panneau comportant le

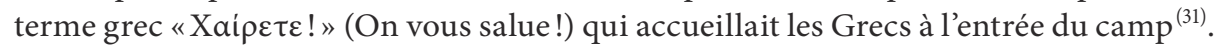

L'un des aspects les plus frappants du séjour des Grecs à Görlitz fut l'intérêt suscité par leur présence au sein de la communauté scientifique allemande, en particulier

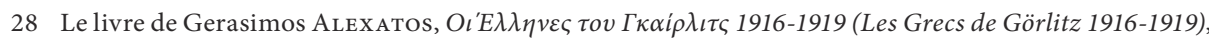
Thessalonique, Kyriakidis, 2010, peut être considéré comme un jalon important de ce renouvellement historique. Très importante également est l'édition récente du témoignage de Stylianos Kandylakis, officier de la gendarmerie interné à Görlitz: Gerasimos Alexatos, Stratos Dordanas, Manolis

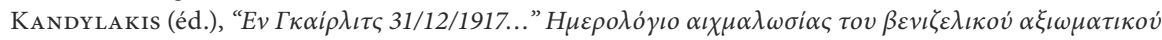

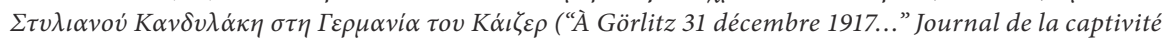
de l'officier vénizéliste Stylianos Kandylakis dans l'Allemagne du Kaiser), Thessalonique, Kyriakidis, 2014. Pour une présentation sommaire des axes principaux de ce témoignage, voir aussi Gerasimos

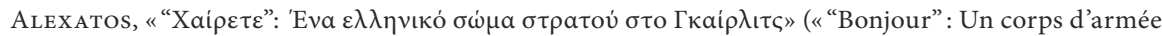
grec à Görlitz», in: Chrysos/Schultheiss (dir.), Les étapes décisives des relations gréco-allemandes (note 10), p. 185-198.

29 G. B. LEON (Leontaritis), Greece and the Great Powers (note 2), p. 380-389, 396-400.

30 G. Alexatos, Les Grecs de Görlitz 1916-1919 (note 28), p. 62-64.

31 Ibid., p. 71-74. 
pour ceux qui s'intéressaient à la Grèce. Des scientifiques issus de nombreuses disciplines arrivèrent dans la petite ville de Silésie afin de connaître les Grecs de plus près et leur offrir leurs services, en particulier dans le domaine de l'apprentissage de la langue allemande. Dans le même temps, intense fut l'intérêt des linguistes qui affluèrent pour exploiter la richesse linguistique grecque, y compris les éléments dialectiques locaux. On ressent une émotion particulière à la lecture des archives concernant le camp car ces dernières témoignent que c'est à Görlitz que furent réalisés quelques-uns des tout premiers enregistrements vocaux de musique grecque, avec notamment le premier enregistrement documenté de bouzouki ${ }^{(32)}$. Grâce à cet engagement, mais aussi à la vie quotidienne et aux relations entre les Grecs et les habitants de la petite ville ${ }^{(33)}$, se constitua un petit groupe de population grecque ayant une bonne connaissance de la langue et de la culture allemandes. Ce groupe, malgré la dissolution et la dispersion d'après-guerre, allait devenir, à travers plusieurs cas individuels, un milieu fructueux pour les relations entre les deux pays dans les décennies à venir.

Par ailleurs, la vie à Görlitz ne pouvait être épargnée par les évolutions politiques et militaires générales, au niveau intérieur comme extérieur. La controverse entre royalistes et vénizélistes, malgré le nombre relativement faible de ces derniers, érodait déjà, depuis le tout début du séjour à Görlitz, la cohésion et la paix au sein de la communauté grecque. Le phénomène fut exacerbé après les "Noemvriana» de 1916 et encore davantage après le détrônement de Constantin à l'été $1917^{(34)}$. Ces tensions aiguës conduisirent les Allemands, malgré les objections de Chatzopoulos, à déplacer, le dernier jour de l'année 1917, un groupe d'officiers vénizélistes vers la ville de Werl (Westphalie), lieu où ils restèrent en détention jusqu'à la fin de la guerre ${ }^{(35)}$. À partir de ce moment, le camp de Görlitz se trouva au centre de l'attention des agents du camp royaliste et le corps d'armée grec devint de plus en plus un outil de négociation, en relation avec les projets concernant son hypothétique intervention dans une opération sur le front des Balkans ${ }^{(36)}$. On peut en outre noter que, depuis juillet 1917, sous le poids des événements qui s'étaient produits à Athènes, avait commencé l'utilisation de soldats grecs pour des travaux rémunérés réalisés dans toute l'Allemagne. C'était une demande des Allemands depuis les tout débuts de la présence grecque, mais elle avait rencontré les objections de Chatzopoulos, qui en craignait notamment les conséquences pour l'état physique de ses hommes ${ }^{(37)}$.

32 Ibid., p. 105-111.

33 Bien que la présence des Grecs dans la petite ville ait eu plusieurs aspects positifs, il y eut quelques difficultés, notamment la difficulté à s'adapter à l'hiver allemand. Une autre difficulté était l'alimentation: d'une part, l'Allemagne connaissait alors une crise alimentaire liée au blocus allié, d'autre part les habitudes alimentaires allemandes étaient très différentes des habitudes alimentaires grecques, ce qui entraîna de graves problèmes de santé chez un certain nombre de Grecs (ibid., p. 122-124).

34 Ibid., p. 148-149.

35 Ibid., p. 149-151. Les notes journalières de Stylianos Kandylakis constituent une source importante pour les conflits entre royalistes et vénizélistes à Görlitz, mais aussi pour la détention à Werl: voir Alexatos/Dordanas/Kandylakis, “À Görlitz 31.12.1917...” (note 28), surtout p. 16-18 et 80-115.

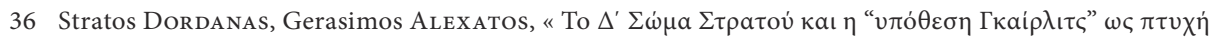

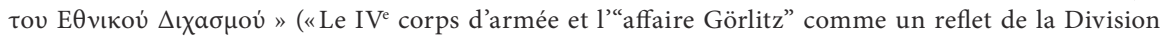
Nationale»), in: Alexatos/Dordanas/Kandylakis (dir.), «À Görlitz 31.12.1917... » (note 28), p. 68-70.

37 G. Alexatos, Les Grecs de Görlitz 1916-1919 (note 28), p. 132-135. 
L'acte final de la présence des Grecs à Görlitz fut causé par la vague révolutionnaire qui balaya une grande partie de l'Allemagne à la fin de 1918. La participation massive des soldats au mouvement spartakiste provoqua des troubles dans le camp, l'expulsion du gouverneur et la mise en place de conseils militaires. Dans le chaos général qui régnait alors en Allemagne, la rupture avec les autorités locales était presque inévitable. Le départ des Grecs et leur retour en Grèce se réalisèrent de manière désordonnée, dans des conditions difficiles, parfois marquées par des incidents violents ${ }^{(38)}$.

L'écho de la présence grecque à Görlitz fut très intense et multiforme en Grèce, y compris plusieurs années après leur départ. Sur la scène nationale, la «captivité » du $I^{\mathrm{e}}$ corps d'armée resta dans l'actualité politique et la nécessité de rendre des comptes sur les événements de septembre 1916 conduisit à la condamnation de plusieurs protagonistes. Parmi ceux qui revinrent du camp de Görlitz, nombreux sont ceux qui furent les vecteurs d'une relation particulière à l'espace germanique. Un certain nombre d'entre eux étaient des hommes de lettres et des artistes qui jouèrent un rôle significatif dans la vie culturelle grecque au $\mathrm{XX}^{\mathrm{e}}$ siècle. Les mariages mixtes avec des femmes allemandes furent aussi une réalité, même si, dans de nombreux cas, le destin de ces couples après leur retour en Grèce ne fut pas toujours heureux ${ }^{(39)}$.

Aujourd'hui est arrivé le temps de l'étude et de l'interprétation de cet événement de manière scientifique. Nous pouvons donc dire que l'internement des militaires grecs à Görlitz fut un épisode important de l'histoire gréco-allemande de la Première Guerre mondiale, épisode qui influença d'une manière particulière l'équilibre déjà délicat des relations bilatérales entre Grèce et Allemagne. Son évolution est inextricablement liée aux évolutions politiques, militaires et diplomatiques des deux pays, mais son étude approfondie doit aller au-delà des limites étroites de l'histoire politique et militaire et utiliser les méthodes et les outils de l'histoire sociale et culturelle, afin de pouvoir être compris dans toutes ses dimensions.

Pour conclure, on dira que l'année 1917 fut une année décisive pour l'issue de la Première Guerre mondiale ${ }^{(40)}$, notamment en relation avec les conditions qui conduisirent aux dernières batailles décisives et aux processus diplomatiques de 1918. Mais cette année fut aussi importante, comme nous l'avons vu dans les pages précédentes, en raison des évolutions concernant la position de la Grèce dans la guerre et ses relations avec l'Allemagne. Quatre points sont, selon nous, à retenir:

- L’année 1917 fut l'année décisive durant laquelle la longue et épineuse question de l'attitude de la Grèce dans la guerre fut définitivement résolue. Après le départ du roi Constantin et le retour au pouvoir de Venizélos, le pays abandonna officiellement sa neutralité pour rejoindre le camp de l'Entente. Parallèlement, toute la période jusqu'au détrônement de Constantin fut caractérisée par la densification des processus diplomatiques autour de la question grecque, l'apogée des interventions étrangères dans les affaires grecques et les difficultés croissantes de la vie quotidienne du peuple grec.

38 Ibid., p. 152-165.

39 HStAS, E 151/02 1090, Document émanant du ministère allemand de l'Intérieur, n I 8105/2.12 du 21 décembre 1932.

40 Jean-Jacques Becker, 1917 en Europe. L’année impossible, Paris, Éditions Complexe, 1997; G. B. DER-

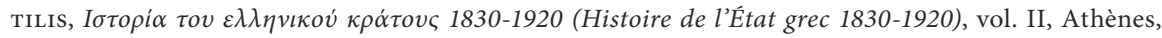
Estia, 2006, p. 893-898. 
- L’adhésion de la Grèce à l'Entente supprima la possibilité d'une attaque coordonnée des forces grecques et des Empires centraux contre l'armée d'Orient sur le front de Macédoine. Au moment, pourtant, où l'Allemagne semblait avoir désormais les mains libres pour satisfaire les exigences de son allié bulgare, la stagnation et l'immobilité sur le front, ainsi que d'autres facteurs, sapèrent progressivement la résistance et la stabilité du camp bulgare, avec des conséquences qui se révélèrent douloureuses pour les Puissances centrales à l'automne de l'année suivante.

- Après l'entrée en guerre de la Grèce, les relations gréco-allemandes se trouvèrent à un tournant, les deux pays appartenant maintenant officiellement à des camps adverses. Le camp allemand durcit sa position envers la Grèce, tout en maintenant des canaux de communication informels avec l'ex-roi exilé en Suisse.

- Ce moment difficile pour les relations bilatérales se refléta également dans la réalité de Görlitz, où étaient installés depuis 1916, sous un statut de «libre captivité», les soldats du IV e corps d'armée grec. Le transfert de groupes de soldats pour travailler dans d'autres parties du territoire allemand fut imposé, tandis qu'à la fin de l'année 1917, on tenta l'épuration du corps des officiers d'obédience vénizéliste et leur incarcération dans la ville de Werl. La situation de ces enclaves grecques singulières au cœur de l'Allemagne reflète la situation politique, diplomatique et militaire de toute la Grèce dans le tournant le plus critique de la Première Guerre mondiale.

\section{Résumé}

1917 a été une année cruciale non seulement pour l'histoire de la Première Guerre mondiale dans son ensemble (et pour le rôle spécifique de la Grèce dans le conflit), mais aussi pour le développement des relations gréco-allemandes dans ce contexte plus large. En termes diplomatiques, cette année a été marquée par l'adhésion de la Grèce au camp de l'Entente, mais aussi par d'autres efforts grecs pour approcher l'Allemagne, menés par le camp pro-allemand autour du roi exilé Constantin. Au niveau militaire, le débat sur un plan éventuel d'une attaque par les puissances centrales contre les forces de l'Entente à Thessalonique a continué à exister, même si cette idée avait été exclue par le leadership militaire allemand au début de l'année; en même temps, les premiers signes de fatigue apparaissaient parmi les Bulgares, un allié clé de l'Allemagne dans la région. Enfin, la présence d'une grande unité militaire grecque dans la ville allemande de Görlitz a donné une nouvelle dimension aux relations germano-grecques, servant de noyau de contacts et de tensions, mais aussi comme un facteur important dans les relations diplomatiques de cette époque.

\section{Zusammenfassung}

1917 war ein entscheidendes Jahr nicht nur für die Geschichte des Ersten Weltkriegs als Ganzes (und für die konkrete Rolle Griechenlands im Konflikt), sondern auch für die Entwicklung der griechisch-deutschen Beziehungen in diesem breiteren Kontext. In diplomatischer Hinsicht war dieses Jahr nicht nur durch den Beitritt Griechenlands zum Entente-Lager geprägt, aber auch von anderen Bemühungen, insbesondere die um dem prodeutschen Lager des verbannten König Konstantin, sich Deutschland zu nähern. Auf militärischer Ebene setzte sich die Debatte über einen eventuellen Angriffsplan der 
Mittelmächte gegen die Entente-Kräfte in Thessaloniki fort, auch wenn diese Idee zu Beginn des Jahres von der deutschen Militärführung ausgeschlossen worden war; gleichzeitig erschienen erste Müdigkeitsanzeichen unter den Bulgaren, eines der Schlüsselalliierten Deutschlands in der Region. Schließlich gab die Präsenz einer großen griechischen Militäreinheit in der deutschen Stadt Görlitz den griechisch-deutschen Beziehungen eine neue Dimension, zugleich Kern von Kontakten und Spannungen, aber auch wichtiger Faktor der diplomatischen Beziehungen dieser Zeit.

\begin{abstract}
1917 was a crucial year not only for the history of World War I as a whole (and for Greece's specific role in the conflict), but also for the development of Greek-German relations within this broader context. In diplomatic terms, this year was marked by the accession of Greece to the Entente camp, but also by further Greek efforts to approach Germany, carried out by the pro-German camp around the exiled King Constantine. At the military level, the debate on an eventual plan of an attack by the Central Powers against the Entente forces in Thessaloniki continued, even if this idea had been ruled out by the German military leadership at the beginning of the year; at the same time, the first signs of fatigue appeared among the Bulgarians, a key ally of Germany in the region. Finally, the presence of a large Greek military unit in the German city of Görlitz gave a new dimension to Greek-German relations, serving as a nucleus of contacts and tensions, but also as an important factor in the diplomatic dealings of that time.
\end{abstract}

